\title{
Physiological based Modeling and Control of an Industrial Tank Process
}

\author{
Svein H. Stokka Kim A. Jakobsen Kristian Thorsen Tormod Drengstig \\ Department of Electrical Engineering and Computer Science, University of Stavanger, Norway, \\ \{sh.stokka, ka.jakobsen\}@stud.uis.no, \{kristian.thorsen, tormod.drengstig\}@uis.no
}

\begin{abstract}
In this paper we make a physiological based dynamic model of a laboratory scale industrial tank process configured with an inlet pump and an outlet valve. Thus, the pump and valve characteristics, and also the functional expression for the hydrostatic pressure, are fitted to enzymatic mechanisms and saturable signaling kinetics. The model is verified against process data and shows good compliance. Based on the model, we tune and apply a physiological inspired control structure to control the water level in the presence of disturbances in the inlet pump speed. This control structure is further compared to an Icontroller, and we show that one of the main properties of physiological control is that the steady state level of the controlled variable may deviate from the setpoint value. We continue by showing how to lump this deviation and the setpoint into a variable setpoint controller. The main benefit of such a controller is reduced variation in the control signal (calculated as total variation, TV). Based on these results, we finally suggest an industrial control structure where the control signal participates in the continuous calculation of a variable setpoint.
\end{abstract}

Keywords: physiological control, integral control, tank process, variable setpoint

\section{Introduction}

\subsection{Physiological and Industrial Control}

Systems biology includes the study of how biochemical species in cells and organisms interact to create control structures. These control structures are often functionally compared to well known man-made industrial control systems such as PIDcontroller (Watson et al., 2011; Ang et al., 2010), feedforward structures (Savageau and Jacknow, 1979; Sontag, 2010; LeDuc et al., 2011), fuzzy control (Ding, 2000), positive feedback (Cloutier and Wang, 2011), robust control (Goulian, 2004), and adaptive control (Javaherian et al., 2009). Thus, some of the biochemical species are considered controlled variables, while other function as manipulated variables. The interaction between the species is termed signalling or signal transduction (Han et al., 2007), and compared to industrial processes these signalling events represents the measurement function and the connection between the controller output and the actuator. Along this path of research, we have in a series of publications investigated biochemical mechanisms and kinetic properties behind integral feedback in physiology (Ni et al., 2009; Drengstig et al., 2012).

In this paper we take an opposite approach and apply physiological inspired control to a laboratory scale industrial tank process, illustrated in Figure $1 a$. The work is an extension of the bachelor's thesis of the first two authors (Stokka and Jakobsen, 2017). Our aim is here to explore the differences between physiological and industrial control, and to identify properties and functions of physiological control that might be useful in an industrial context.

In the literature, there are not many reports showing physiologically inspired control structures applied to industrial processes. One example, however, is found in (Pérez-Correa et al., 2015) where a repressor Hill function representing the proportional part, together with standard integrative part, are applied to control the level of a conic tank.

\subsection{Controller Motifs}

A biochemical network with regulatory properties must in its simplest form include at least two components or state variables, i.e. one controlled component and one controller component. The controller component acts on the controlled component in a way that it compensates for external disturbances, and thereby represents a negative feedback. We have earlier presented a collection of simple two-component regulatory networks (Drengstig et al., 2012), and we have used the name controller motifs to describe them. These motifs consist of two chemical species, $A$ and $E$, both of them being formed and turned over. $A$ may represent an intracellular compound which is subject to disturbances in the form of e.g. uncontrolled diffusive transport of $A$ in and out of the cell, and $E$ may represent a membrane bound compound such as a transporter protein. This is illustrated in Figures $1 \mathrm{~b}$ and 1c.

So far, we have identified the eight different controller motifs shown in Figure 2. Based on the controller action, i.e. whether the $E$ mediated compensatory flow is located upstream or downstream of $A$, these controller motifs are further classified as either inflow or outflow controllers with activating or inhibiting control actions (Figure 2). 
$a$
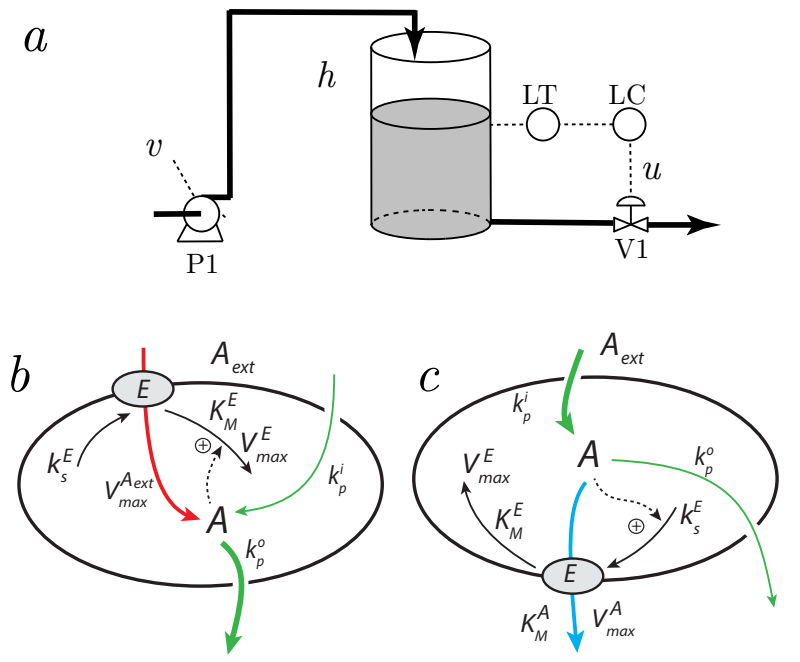

Figure 1. Panel $a$ : Illustration of the laboratory scale industrial tank process used in this work. The level in the tank is maintained by the level controller manipulating the outlet valve in the presence of disturbances $v$ (pump speed). Panels $b$ and $c$ : Illustration of a cell with a compound $A$ being under homeostatic control by an inflow controller (panel $b$ ) or an outflow controller (panel $c$ ). Panel $b$ : An inflow controller compensate for outflow perturbations, $k_{p}^{o}$ (thick green line), in $A$ by adding more $A$ through an $E$-mediated inflow (red line). Panel $c$ : An outflow controller compensate for inflow perturbations, $k_{p}^{i}$ (thick green line), in $A$ by removing excess of $A$ through an $E$-mediated outflow (blue line).

\section{Modeling}

Applying the mass balance to the tank process in Figure $1 a$ give the following differential equation describing the dynamics of the water level $h$ :

$$
\dot{h}=\frac{1}{A_{\operatorname{tank}}}\left(f_{P 1}(v)-\beta \cdot f_{V 1}(u) \cdot \sqrt{h}\right)
$$

where $A_{\text {tank }}$ is the area of the tank, $f_{P 1}(v)$ and $f_{V 1}(u)$ are the pump and valve characteristics, $\sqrt{h}$ is the influence of the hydrostatic pressure on the outlet flow, and $\beta$ is a constant comprising density $\rho$, gravity $g$, valve flow coefficient $K_{v}$, and unit conversion factors. In order to represent the tank model in Eq. (1) as one of the motifs in Figure 2, we note first that the tank process is configured as an outflow controller. Thus, there are four possible candidates, i.e. outflow controller 5 to 8 . Since the valve V1 is normally closed, there is a direct relationship between the control signal and the valve opening, which corresponds to activating kinetics ${ }^{1}$. The pump P1 is at rest when the disturbance signal $v$ is zero, and hence, this also represents activating kinetics. The tank process can therefore be described as an outflow controller 5 motif.

Based on the non-linearity of the functions $f_{P 1}(v)$, $f_{V 1}(u)$, and $\sqrt{h}$, all shown as solid lines in Figure 3, we express them as general Hill kinetic expressions in the physiological model. Thus, the physiological model

\footnotetext{
${ }^{1}$ Thus, a normally open valve correspond to inhibiting kinetics.
}

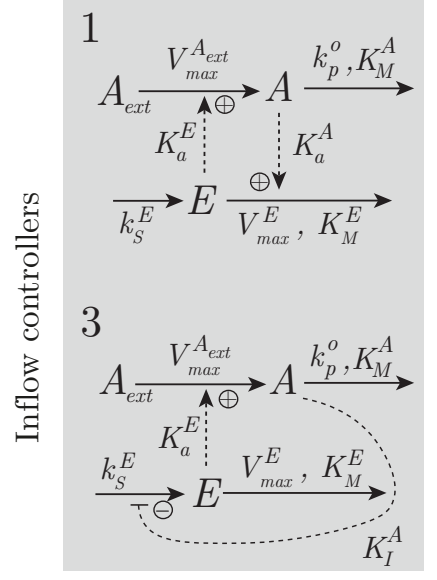

2

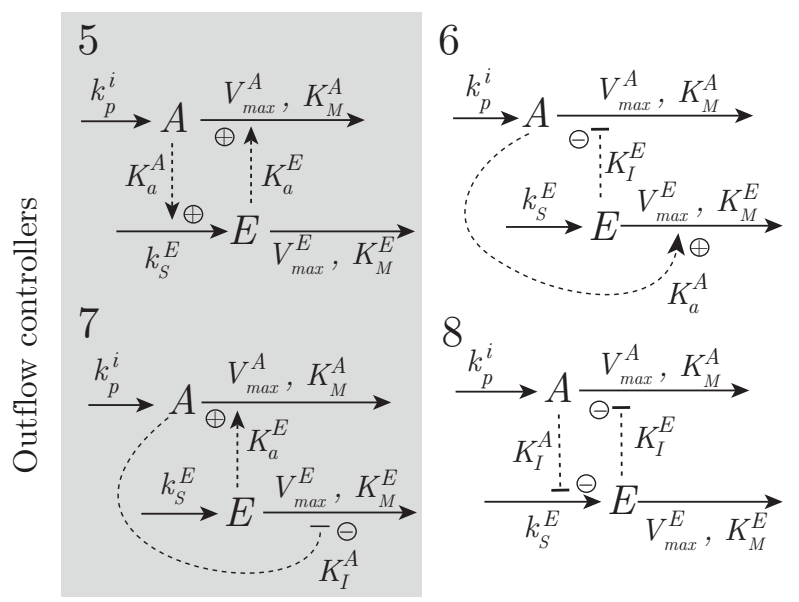

Figure 2. Inflow and outflow controller motifs with saturable activating action (gray background) or inhibiting action (white background). The controlled species $A$ is subject to outflow or inflow perturbations $\left(k_{p}^{o}\right.$ or $\left.k_{p}^{i}\right)$. The controller species $E$ compensates for this perturbation through an $E$-mediated inflow or outflow of $A$, respectively. The synthesis of $E$ is modeled with a rate konstant $k_{s}^{E}$, whereas the degradation $E$ is assumed to be a first order Hill kinetics with a saturation constant $K_{M}^{E}$. Similar saturable enzymatic reactions are also assumed in the degradation of controlled species $A$.

written in terms of $A, E$ and $A_{\text {ext }}$ (corresponding to $h, u$ and $v$ ) is shown in Eq. (2). Note that we use Hill coefficients $\{n, m, p\} \in \mathbb{N}^{+}$in order to resemble enzymatic binding sites.

$$
\dot{A}=\frac{k_{p}^{i} \cdot A_{\text {ext }}{ }^{n}}{\left(K_{M}^{A_{e x t}}\right)^{n}+A_{\text {ext }}{ }^{n}}-\frac{V_{\max }^{A} \cdot A^{p}}{\left(K_{M}^{A}\right)^{p}+A^{p}} \cdot \frac{E^{m}}{\left(K_{a}^{E}\right)^{m}+E^{m}}
$$

From comparing Eq. (1) and Eq. (2), we identify the following three relationships:

$$
\begin{aligned}
\frac{1}{A_{\mathrm{tank}}} \cdot f_{P 1}(v) & =\frac{k_{p}^{i} \cdot A_{\text {ext }}{ }^{n}}{\left(K_{M}^{A_{\text {ext }}}\right)^{n}+A_{\text {ext }}{ }^{n}} \\
\frac{1}{A_{\mathrm{tank}}} \cdot \beta \cdot f_{V 1}(u) & =\frac{V_{\max }^{A} \cdot E^{m}}{\left(K_{a}^{E}\right)^{m}+E^{m}} \\
\sqrt{h} & =\frac{A^{p}}{\left(K_{M}^{A}\right)^{p}+A^{p}}
\end{aligned}
$$



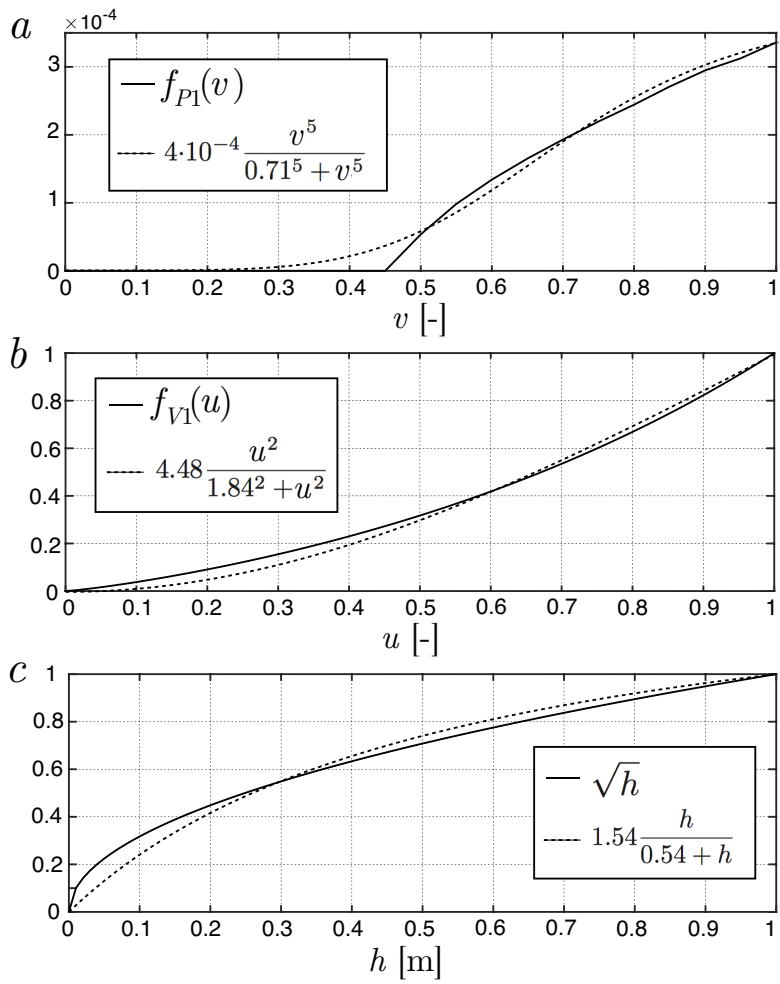

Figure 3. Least squares parameter fitting of model characteristics. Panel $a$ : Pump characteristic $f_{P 1}(v)$ fitted to a Hill kinetic expression with cooperativity of $n=5$. Note that we use a centrifugal pump placed approximately 3 meters below the tank inlet, which implies that there is no water delivery until the pump signal is $v>0.45$. Panel $b$ : Valve characteristic $f_{V 1}(u)$ fitted to a Hill kinetic expression with cooperativity of $m=2$. Panel $c$ : Fitting of $\sqrt{h}$ to Hill kinetic expression with cooperativity of $p=1$. The height of the tank is $1 \mathrm{~m}$.

The least squares estimation of $f_{P 1}(v), f_{V 1}(u)$ and $\sqrt{h}$ are shown as dashed lines in Figure 3. The bottom area of the laboratory tank is $A_{\mathrm{tank}}=0.01 \mathrm{~m}^{2}(10 \times 10 \mathrm{~cm})$ and the valve flow coefficient is $K_{v}=11.25 \mathrm{~m}^{3} /(\mathrm{h} \sqrt{\text { bar }})$. Using $\rho=1000 \mathrm{~kg} / \mathrm{m}^{3}$ and $g=9.8 \mathrm{~m} / \mathrm{s}^{2}$, we find $\beta=9.78 \cdot 10^{-4} \mathrm{~m}^{3} /(\mathrm{s} \sqrt{\mathrm{m}})$. Thus, the first principles based model in Eq. (1) can be written as the physiological based model in Eq. (6):

$$
\dot{h}=\frac{0.04 \cdot v^{5}}{0.71^{5}+v^{5}}-\frac{0.71 \cdot u^{2}}{1.84^{2}+u^{2}} \cdot \frac{h}{0.54+h}
$$

The models in Eqs. (1) and (6) are in Figure 4 verified against real data from the tank process, and we see that both models displays good compliance with real data. Thus, it should be possible to tune a physiological controller for the tank process.

\section{Tuning of an Integral Controller}

We have previously shown that the motifs in Figure 2 share similarities with an integral controller (Drengstig et al., 2012). In order to have a base for comparison when applying physiological inspired

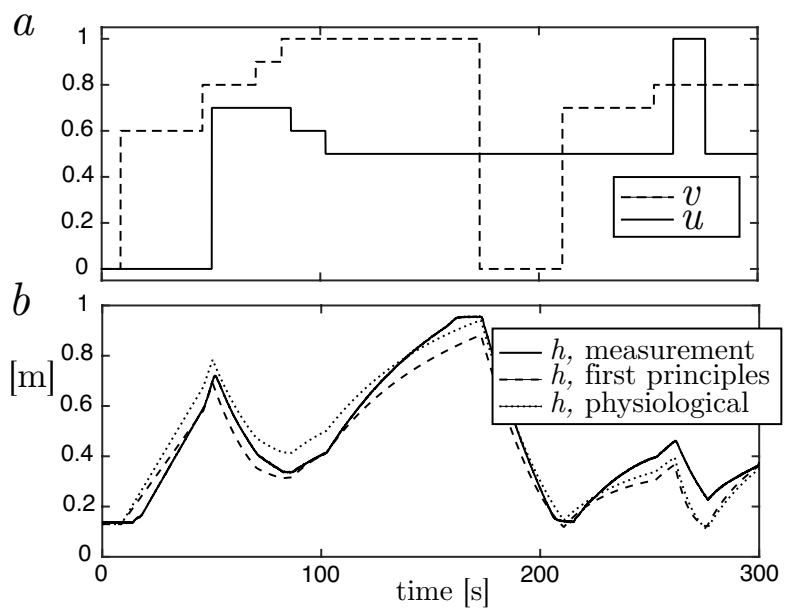

Figure 4. Model verification. Panel $a$ : Valve control signal $u$ (solid line) and pump signal $v$ (dashed line) as a function of time. Panel $b$ : Measured water level in tank (solid line), first principles model in Eq. 1 (dashed line) and physiological model in Eq. 6 (dotted line).

control, we will in this section tune the standard integral controller

$$
u=-G_{i} \int_{0}^{t}\left(h_{s e t}-h\right) d \tau
$$

using the pole-placement method. Note that the gain is negative since the controller is reverse-acting. We use the following experimentally found working point $W P$

$$
W P: v_{0}=0.62, u_{0}=0.32, h_{0}=0.50
$$

and estimate from the model in Eq. (1) the following transfer function for the tank process

$$
H_{p}(s)=\frac{-4.5}{86 s+1}
$$

The closed loop transfer function is then readily found as

$$
M(s)=\frac{G_{i} \cdot 4.5}{86 s^{2}+s+G_{i} \cdot 4.5}
$$

Since we have only a single controller parameter, we cannot specify both the natural undamped frequency $\omega_{n}$ and the damping ratio $\zeta$ of $M(s)$. We consider overshoot to be the most important aspect of the step response, and thus, specify an underdamped response with $50 \%$ overshoot, corresponding to $\zeta=0.2$. Consequently, we find $\omega_{n}=0.029$ and an integral gain of $G_{i}=0.016$.

\section{Tuning of Physiological Controllers}

For a physiological regulatory system to be functional from a control theoretic point of view, it needs a setpoint. In contrast to an industrial control system where the setpoint represents an external input signal, setpoints in physiology are an inherent part of the regulatory mechanism. Even though the biochemical mechanisms behind setpoints in physiology is still not well understood, the notion 
of a fixed setpoint has so far been dominating (Ma et al., 2009; Drengstig et al., 2012; Ang and McMillen, 2013). This implies, however, that there is often a need for a deviation measure to count for the fact that in many cases the steady state value of the controlled variable deviates from, what is believed to be, the setpoint value. An alternative way of interpreting this deviation is to consider a variable setpoint, and we will in the next two subsections investigate both approaches.

\subsection{Fixed Setpoint Approach}

The controller part of motif 5 is from Figure 2 generally given as

$$
\dot{E}=k_{s}^{E} \cdot \frac{A}{K_{a}^{A}+A}-\frac{V_{\max }^{E} \cdot E}{K_{M}^{E}+E}
$$

where $K_{a}^{A}$ is the activation constant. The basis for the fixed setpoint approach is the assumption that the enzyme which degrades the controller species $E$ is operating under saturated conditions, i.e. $K_{M}^{E} \ll E$. An example of where such an approximation is valid is the degradation of alcohol by the enzym alcohol dehydrogenase, where the degradation rate is independent of the alcohol content in blood.

In Eq. (11), the information about the level of $A$, i.e. the signaling between $A$ and $E$, is based on mixed activation kinetics. However, the water level measurement in the tank process is a linear function of water level $h$, i.e. $K_{a}^{A}=0$. Thus, using variable names from the tank process ( $E=u, A=h)$, we write Eq. (11) as

$$
\dot{u}=k_{s}^{u} \cdot h-\frac{V_{\max }^{u} \cdot u}{K_{M}^{u}+u}
$$

which can be restructured as the integral control law in Eq. (13)

$$
\dot{u}=-\underbrace{k_{s}^{u} \cdot \frac{u}{K_{M}^{u}+u}}_{G_{i}} \cdot(\underbrace{\frac{V_{\max }^{u}}{k_{s}^{u}}}_{h_{\text {set }}}-\underbrace{\frac{K_{M}^{u}+u}{u} \cdot h}_{h_{\text {meas }}})
$$

Here $G_{i}$ is still the controller gain and $h_{\text {set }}$ is the fixed setpoint consisting of parameter values only. We note that the controller gain $G_{i}$ is affected by the control signal $u$, and we observe also the premise for the necessary condition behind the fixed setpoint approach, i.e. $K_{M}^{u} \ll u$. If this condition is satisfied, the fraction $\frac{K_{M}^{u}+u}{u}$ acting on the measurement function becomes close to unity, and can hence be discarded. A visualization of Eq. (13) is shown in Figure $5 a$, which illustrates that the control signal $u$ will have a (small) influence on the measurement $h_{\text {meas }}$ and the controller gain.

From Eq. (13) we note that $k_{s}^{u}$ is a part in both the controller gain $G_{i}$ and the setpoint $h_{\text {set }}$. Thus, tuning the controller around a fixed setpoint implies that both parameters $k_{s}^{u}$ and $V_{\max }^{u}$ must be adjusted in order to maintain the setpoint. Taking advantage of the controller tuning in the
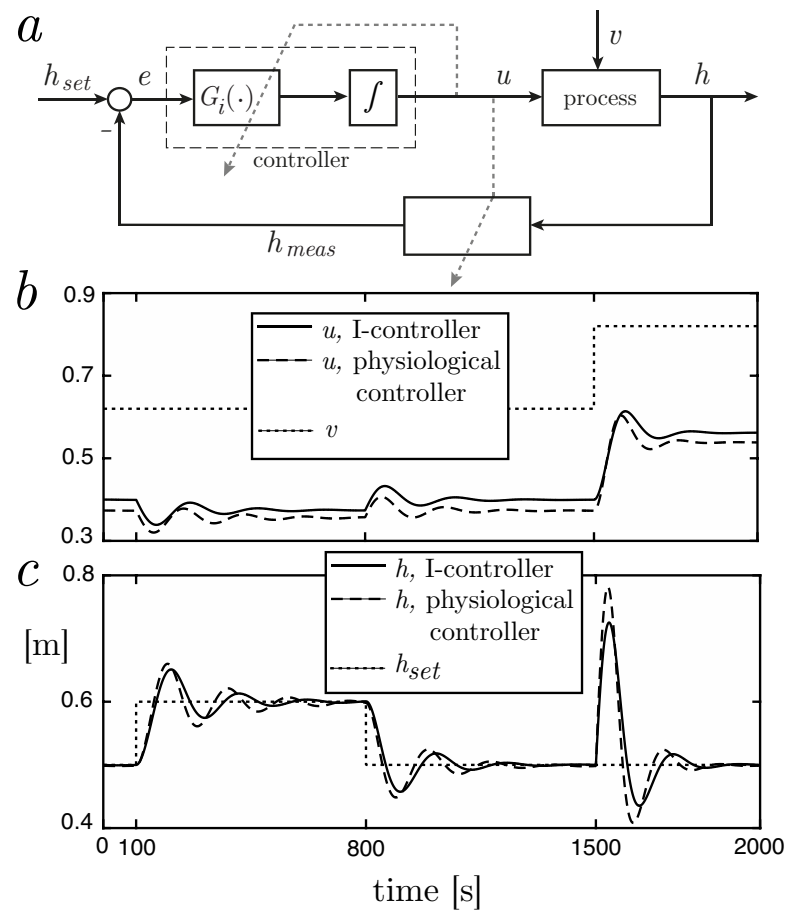

Figure 5. Panel $a$ : Negative feedback loop for the fixed setpoint approach, where the setpoint depends only on parameter values. Panels $b$ and $c$ : Comparing the setpoint tracking and disturbance rejection properties of $i$ ) the first principles based model in Eq. (1) together with the I-controller in Eq. (7) (solid lines), and $i$ ) the physiological model in Eq. (6) together with the controller in Eq. (12) (dashed lines). Panel $b$ : Stepwise increase in disturbance $v$ from 0.62 to 0.82 at $t=1500$ s (dotted line) and corresponding manipulated variables $u$ (solid and dashed lines). Panel $c$ : Response in water level $h$ for a setpoint change from 0.5 to $0.6 \mathrm{~m}$ at $t=100 \mathrm{~s}$ and back again at $t=800 \mathrm{~s}$. The response also shows the disturbance rejection properties for the step in $v$ at $t=1500 \mathrm{~s}$. The setpoint change for the physiological controller is caused by a $20 \%$ increase in $V_{\max }^{u}$. Parameter values for the physiological controller are $V_{\max }^{u}=0.008, k_{s}^{u}=0.016$, and $K_{M}^{u}=0.001$. Controller gain for the integral controller is $G_{i}=0.016$.

previous section, we specify $k_{s}^{u}=G_{i}=0.016$, and hence, in order to have a setpoint of $h_{\text {set }}=0.5 \mathrm{~m}$, we get $V_{\max }^{u}=0.008$.

We have in Figure 5, panels $b$ and $c$, simulated the setpoint tracking and disturbance rejection properties of

i) the first principles based model in Eq. (1) together with the I-controller in Eq. (7) (solid lines), and

ii) the physiological model in Eq. (6) together with the physiological controller in Eq. (12) (dashed lines).

We note that both control systems display similar responses to both setpoint changes and stepwise disturbances in $v$. The difference between the control systems is due to the use of different process models and to the value of $K_{M}^{u}$ (even though it is small). 


\subsection{Limitations of the Fixed Setpoint Ap- proach}

If the assumption of saturated removal of the controller species does not hold, i.e. $K_{M}^{u} \nless u$, there will be a deviation between the fixed setpoint and the actual water level. Thus, the fraction $\frac{K_{M}^{u}+u}{u}$ is not close to unity, and con-
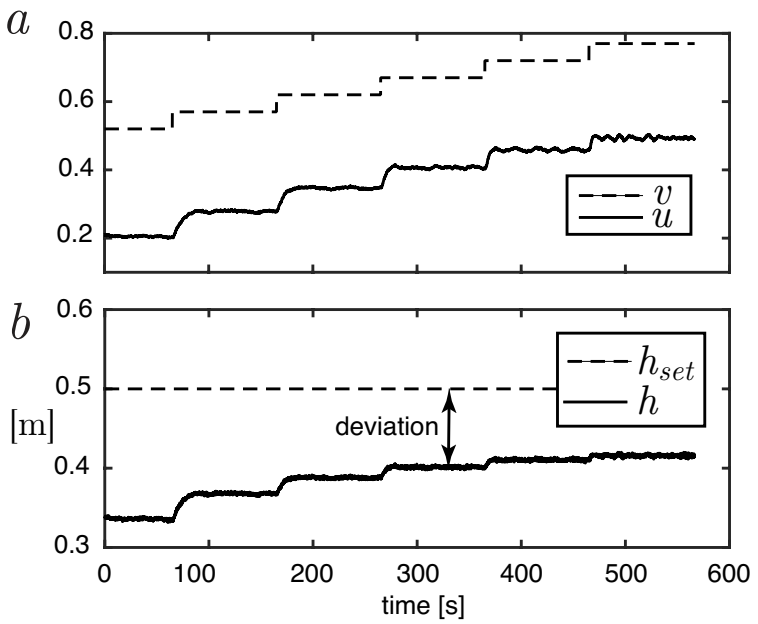

Figure 6. Physiological control of the tank process demonstrating the deviation between the fixed setpoint and the actual water level when $K_{M}^{u} \nless u$. The tank is controlled by the controller in Eq. (12) with $K_{M}^{u}=0.1$. In order to avoid oscillatory behaviour the controller gain is increased to $k_{s}^{u}=10$, giving $V_{\max }^{u}=5$ for a fixed setpoint of $h_{\text {set }}=0.5 \mathrm{~m}$. Panel $a$ : The pump speed $v$ is increased in a stepwise manner from $v=0.52$ to $v=0.77$ (dashed line), together with the corresponding control signal $u$ (solid line). Panel $b$ : Measured water level and the fixed setpoint. Note that the deviation decreases as $v$ (and $u$ ) increases.

sequently the water level $h$ will deviate from the setpoint $h_{\text {set }}$ (even though the control error $e=0$ ). This behaviour is shown in Figure 6, which shows the results from an experiment applied to the tank process where we use $K_{M}^{u}=0.1$ instead of $K_{M}^{u}=0.005$ as in Figure 5. We observe that the deviation decreases as the control signal $u$ increases, which motivates for a variable setpoint description.

\subsection{Variable Setpoint Approach}

Many physiological systems display variability in regulated species. Examples include blood glucose level as a function of constant glucose infusion (Elrick et al., 1964), and sodium content as a function of salt intake (Bonventre and Leaf, 1982). Such a behavior fits a variable setpoint regulatory system, which can mathematically be found by reorganizing Eq. (12) as follows:

$$
\dot{u}=-\underbrace{k_{s}^{u}}_{G_{i}} \cdot(\underbrace{\frac{V_{\max }^{u}}{k_{s}^{u}} \cdot \frac{u}{K_{M}^{u}+u}}_{h_{\text {set }}}-h)
$$

A visualization of this feedback structure is given in Figure $7 a$, and we note that $u$ now affects the setpoint, rather than the measurement function and controller gain as in Figure $5 a$. In effect, this structure lumps the fixed setpoint and the deviation into a variable setpoint, and consequently adds the variation in the controlled variable $h$ as an addition feature in the controller tuning.

We observe from Figure 6 that as the disturbance $v$ increases, both the control input $u$ and water level $h$ increases. Thus, in order to obtain a control system with a specified variability in the water level $h$, we introduce the notion of a low and a high level of each variable, defined by the working points $W P_{1}$ and $W P_{2}$ as follows:

$$
W P_{1}: v_{l o w}, u_{l o w}, h_{l o w}, W P_{2}: v_{\text {high }}, u_{\text {high }}, h_{\text {high }}
$$

In an industrial application the levels $h_{\text {low }}$ and $h_{h i g h}$ would then correspond to the variation we consider as acceptable at the correlated levels of disturbances, i.e. $v_{l o w}$ and $v_{\text {high }}$. Thus, the tuning of the physiological controller, i.e. determining the three parameters $k_{s}^{u}, V_{\text {max }}^{u}$ and $K_{M}^{u}$, is based on the following two steady state versions of Eq. (14)

$$
\begin{aligned}
& 0=-k_{s}^{u} \cdot\left(\frac{V_{\text {max }}^{u}}{k_{s}^{u}} \cdot \frac{u_{\text {low }}}{K_{M}^{u}+u_{\text {low }}}-h_{\text {low }}\right) \\
& 0=-k_{s}^{u} \cdot\left(\frac{V_{\text {max }}^{u}}{k_{s}^{u}} \cdot \frac{u_{\text {high }}}{K_{M}^{u}+u_{\text {high }}}-h_{\text {high }}\right)
\end{aligned}
$$

As this in an underspecified system, one of the parameters must be specified. This could typically be the controller gain, i.e. the synthesis rate $k_{s}^{u}$ in Eq. (14).

We will illustrate the concept by tuning two different variable setpoint controllers and compare them for a stepwise increase in the disturbance from $v_{l o w}$ to $v_{\text {high }}$, while the water level varies between the pre-specified values $h_{\text {low }}$ and $h_{\text {high }}$. Both control systems are initially in the working point $W P_{1}$ corresponding to Eq. (8), i.e.

$$
W P_{1}: v_{\text {low }}=0.62, u_{\text {low }}=0.32, h_{\text {low }}=0.50
$$

We then specify the high level disturbance as $v_{\text {high }}=0.82$ together with the two different high water levels indicated with gray background in Table 1. Based on these specifications, we find from experiments on the tank process the corresponding two high level control signals $u_{\text {high }}$, also shown in Table 1.

Table 1. Controller parameters calculated for the given $W P_{1}$ in Eq. (18) and two different $W P_{2}$.

\begin{tabular}{|c||c|c||c|c|c|}
\hline \multirow{2}{*}{$\begin{array}{c}\text { Controller } \\
\text { no. }\end{array}$} & \multicolumn{2}{|c||}{$W P_{2}, v_{\text {high }}=0.82$} & \multicolumn{4}{c|}{ Parameters } \\
\cline { 2 - 6 } & $h_{\text {high }}$ & $u_{\text {high }}$ & $k_{s}^{u}$ & $V_{\max }^{u}$ & $K_{M}^{u}$ \\
\hline$i)$ & 0.55 & 0.49 & 0.01 & 0.0071 & 0.137 \\
$i i)$ & 0.66 & 0.47 & 0.01 & 0.0177 & 0.797 \\
\hline
\end{tabular}

As we have one degree of freedom in selecting controller parameters, we apply an identical controller gain $k_{s}^{u}=0.01$ for both controllers, and solve Eqs. (16) and (17) to determine $V_{\text {max }}^{u}$ and $K_{M}^{u}$ as shown in Table 1. 
In the simulation in previous subsection (Figure 5), we applied both a setpoint change and a step in the disturbance. In this subsection, however, the setpoint enters the control system as user specified high and low water levels during normal operation, and it is therefore counterintuitive to apply a setpoint change as in Figure 5. Thus, the main task of a variable setpoint regulatory system is therefore to compensate for the disturbances. As this is also the case for many industrial control systems, we compare only the disturbance rejection properties of the two control systems. For this task, we calculate the total variation (TV) as a quantitative measure of controller performance, i.e.

$$
\mathrm{TV}=\sum|u(k)-u(k-1)|
$$

Both physiological controllers are applied to the tank process in Figure $1 a$, and the results are shown in Figure 7 , panels $b-d$. The disturbance is given as a step from $v_{\text {low }}=0.62$ to $v_{\text {high }}=0.82$ at $t=200$ seconds (not shown).

Even though the controller gain for both controllers are the same $\left(k_{s}^{u}=0.01\right)$, we observe that the respons in $h$ is less oscillatory for the controller with the highest acceptable water level $h_{\text {high }}$ (controller $\left.i i\right)$ ). This observation is verified by the lowest total variation (TV) measure in panel $d$. From a physiological point of view, such a reduced control signal usage will save energy in that the
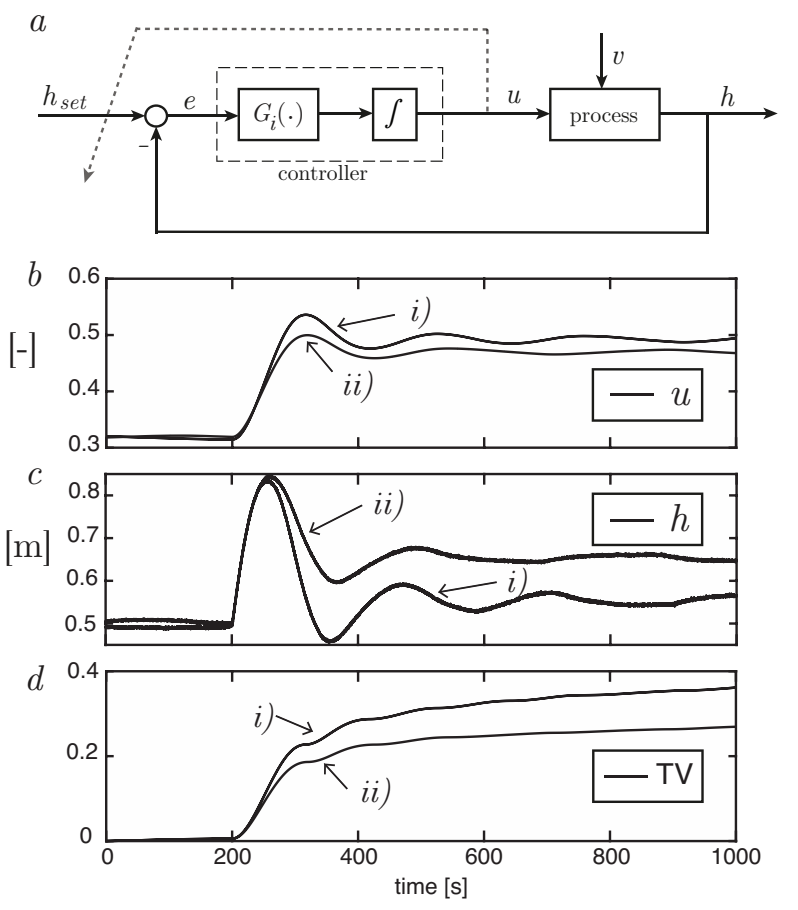

Figure 7. Panel $a$ : Illustration of the negative feedback loop for a variable setpoint controller, where the control signal $u$ have an impact on the setpoint. Panels $b-d$ : Disturbance rejection properties of the two controllers in Table 1 applied to tank process in Figure $1 a$. The responses are numbered according to Table 1. A step in the disturbance from $v_{\text {low }}=0.62$ to $v_{\text {high }}=0.82$ (data now shown) is applied at $t=200$. Panel $b$ : Control input $u$. Panel $c$ : Water level $h$. Panel $d$ : The performance measure total variation (TV) calculated as Eq. (19). variation in synthesis and degradation of the manipulated species $E$ is minimized.

The rationale behind the reduced TV-measure on the tank process is that an increased acceptable high water level $h_{\text {high }}$ leads to increased hydrostatic pressure. This again reduces the necessary compensating valve motion in the outlet flow (compared to a fixed setpoint situation). If such a control system should be applicable in the process industry, it is of vital importance that product quality and safety issues is not affected by the variable setpoint.

\section{An Industrial Control Structure In- spired by Physiology}

Based on the underlying structure behind the variable setpoint controller and the results from the previous subsection, we propose in this section a novel industrial control structure applicable for the process industry. It is based on the feedback loop in Figure $7 a$, and is shown in Figure $8 a$.

The block termed SP-calculation in Figure $8 a$ is where the variable setpoint is continuously calculated. Using two different working points as defined in Eq. (15), the variable setpoint can be calculated as follows

$$
h_{\text {set }}=\frac{\Delta h}{\Delta u} \cdot u+\beta
$$

where $\Delta h=h_{\text {high }}-h_{\text {low }}, \Delta u=u_{\text {high }}-u_{\text {low }}$, and $\beta=h_{\text {low }}-$ $\frac{\Delta h}{\Delta u} \cdot u_{\text {low }}$. The expression in Eq. (20) is just the line going through $\left(u_{\text {low }}, h_{\text {low }}\right)$ to $\left(u_{\text {high }}, h_{\text {high }}\right)$ in a 2 -dimensional space. In order to ensure that the water level is kept between some absolute minimum and maximum limits, the $S P$-calculation-block could also include the necessary logic for this.

In order to quantify the effect of this variable setpoint control structure, we have in a simulation study used $i$ ) a standard PI-controller and $i$ ) a PI-controller with a variable setpoint, both applied to the model in Eq. (1). Both controllers use identical control parameters, i.e. $K_{p}=-1.5$ and $T_{i}=15$. Using the model in Eq. (1), we have specified/calculated the following working points:

$$
\begin{aligned}
& -W P_{1}: v_{\text {low }}=0.55, u_{\text {low }}=0.33, h_{\text {low }}=0.44 \\
& -W P_{2}: v_{\text {high }}=0.80, u_{\text {high }}=0.52, h_{\text {high }}=0.58
\end{aligned}
$$

The responses in the controls signal $u$, water level $h$, and calculated TV are shown in Figures $8 c-e$. As expected, the control signal usage is reduced for the variable setpoint controller. Note that the disturbance shown in Figure $8 b$ has a wider range compared to the working points.

\section{Conclusions}

In this paper we have modelled a tank process, configured with a pumpe and an outlet valve, from a physiological perspective. Both the pump and valve characteristics are fitted to Hill kinetic expressions, and the model is verified against real process data.

We have developed a physiological inspired control system (with a fixed setpoint) and shown that it behaves similar to an integral controller (I-controller). Motivated by 

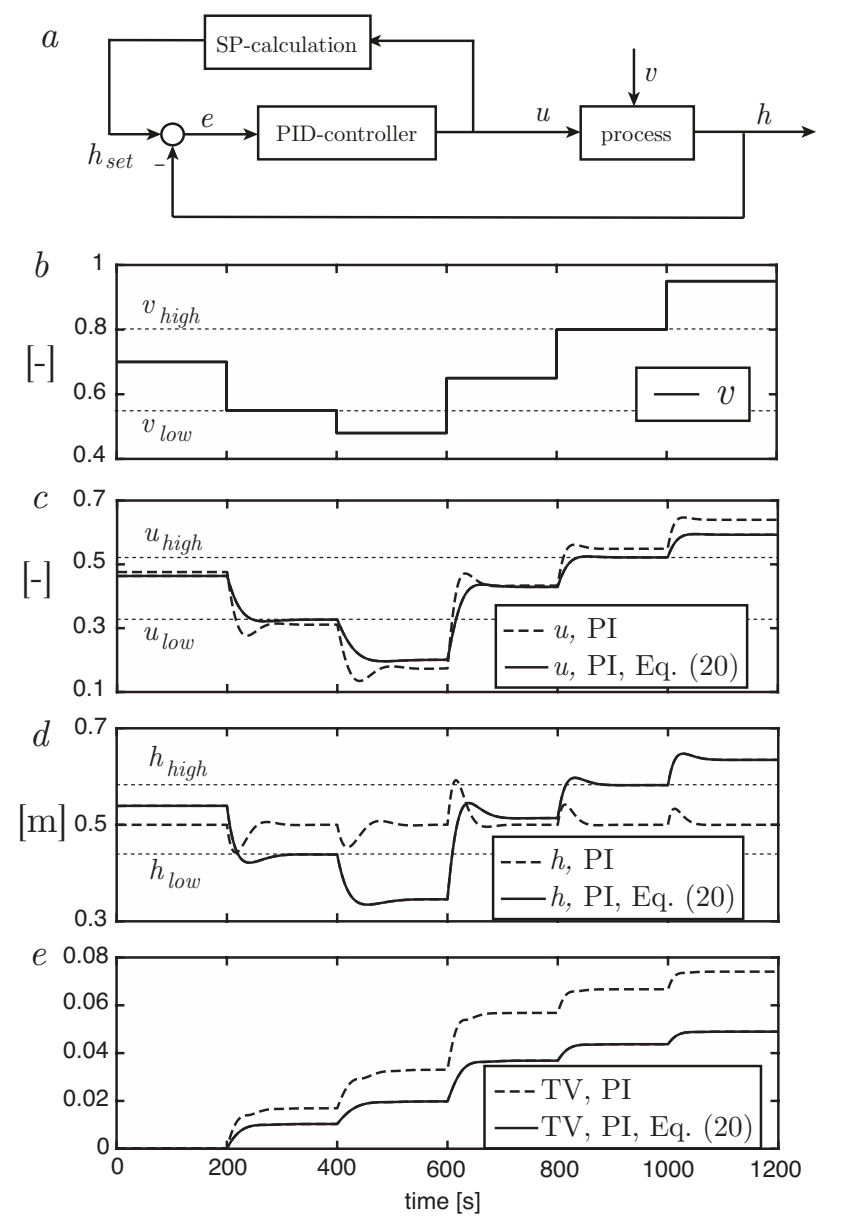

Figure 8. Panel $a$ : Illustration of the negative feedback loop for an industrial control system using a variable setpoint. Panels $b-e$ : Disturbance rejection properties of a standard PI-controller (dashed lines) and a PI-controller with variable setpoint (solid lines). The working points $W P_{1}$ and $W P_{2}$ are shown as dotted lines. Panel $b$ : Disturbance $v$. Panel $c$ : Control input $u$. Panel $d$ : Water level $h$. The setpoint for the standard PI-controller is $h_{\text {set }}=0.5 \mathrm{~m}$. Panel $e$ : The performance measure total variation (TV) calculated as Eq. (19).

the fact that such a fixed setpoint controller is characterized by some deviation measure, we have further shown how this correspond to a control system with a variable setpoint. The benefit of such a structure is that the control signal usage is reduced when the user-specified acceptable variation in the controlled variable increases.

Inspired by this physiologically motivated variable setpoint structure, we finally propose an industrial control structure using a PI(D)-controller with a variable setpoint calculated as a function of the control signal $u$. Simulations indicate that this structure has advantages with respect to control signal usage, compared to a standard PI(D)-controller with an externally given fixed setpoint.

\section{References}

Jordan Ang and David R. McMillen. Physical Constraints on Biological Integral Control Design for Homeostasis and Sen- sory Adaptation. Biophysical Journal, 104(2):505-515, January 2013.

Jordan Ang, Sangram Bagh, Brian P. Ingalls, and David R. McMillen. Considerations for using integral feedback control to construct a perfectly adapting synthetic gene network. Journal of Theoretical Biology, 266(4):723-738, October 2010.

J. V. Bonventre and A. Leaf. Sodium homeostasis: steady states without a set point. Kidney International, 21(6):880-883, 1982.

Mathieu Cloutier and Edwin Wang. Dynamic modeling and analysis of cancer cellular network motifs. Integrative Biology, 3(7):724, 2011.

Yongsheng Ding. A nonlinear PID controller based on fuzzytuned immune feedback law. In Conf Proc Intelligent Control and Automation, pages 1576-1580, 2000.

T. Drengstig, I. W. Jolma, X. Y. Ni, K. Thorsen, X. M. Xu, and P. Ruoff. A Basic Set of Homeostatic Controller Motifs. Biophysical Journal, 103:2000-2010, October 2012.

H. Elrick, L. Stimmler, C. J. Hlad, and Y. Arai. Plasma Insulin Response to Oral and Intravenous Glucose Administration. Journal of Clinical Endocrinology \& Metabolism, 24:10761082, September 1964.

M. Goulian. Robust control in bacterial regulatory circuits. Current Opinion in Microbiology, 7:198-202, April 2004.

Zhangang Han, Thomas M. Vondriska, Ling Yang, W. Robb MacLellan, James N. Weiss, and Zhilin Qu. Signal transduction network motifs and biological memory. Journal of Theoretical Biology, 246(4):7-7, June 2007.

H. Javaherian, Ting Huang, and Derong Liu. A biologically inspired adaptive nonlinear control strategy for applications to powertrain control. In Proc. IEEE Systems, Man and Cybernetics, pages 2076-2082, 2009.

Philip R. LeDuc, William C. Messner, and John P. Wikswo. How do control-based approaches enter into biology? Annu Rev Biomed Eng, 13:369-396, August 2011.

Wenzhe Ma, Ala Trusina, Hana El-Samad, Wendell A. Lim, and Chao Tang. Defining Network Topologies that Can Achieve Biochemical Adaptation. Cell, 138:760-773, August 2009.

Xiao Yu Ni, Tormod Drengstig, and Peter Ruoff. The Control of the Controller: Molecular Mechanisms for Robust Perfect Adaptation and Temperature Compensation. Biophysical Journal, 97:1-11, August 2009.

José Ricardo Pérez-Correa, Gastón Lefranc, and Mario Fernández-Fernández. A New Application of the Hill Repressor Function: Automatic Control of a Conic Tank Level and Local Stability Analysis. Mathematical Problems in Engineering, 2015:1-6, 2015.

M.A. Savageau and G. Jacknow. Feedforward inhibition in biosynthetic pathways: Inhibition of the aminoacyl-tRNA synthetase by intermediates of the pathway. Journal of Theoretical Biology, 77:405-425, 1979. 
E.D. Sontag. Remarks on feedforward circuits, adaptation, and pulse memory. IET Syst Biol, 4(1):39-51, January 2010.

S. H. Stokka and K. A. Jakobsen. Fysiologiske reguleringsmekanismer anvendt på tankprosess. B.Sc. thesis, University of Stavanger, 2017. In Norwegian.

E.M Watson, M.J. Chappell, F. Ducrozet, S.M. Poucher, and J.W. T. Yates. A new general glucose homeostatic model using a proportional-integral-derivative controller. Computer Methods and Programs in Biomedicine, 102:119-129, May 2011. 